J. Gastroenterol. Hepatol. Erkr. 2019 · 17:75-81 https://doi.org/10.1007/s41971-019-00058-1 Online publiziert: 1. Oktober 2019

(c) Der/die Autor(en) 2019

\section{Rudolf Stauber}

Univ.-Klinische Abteilung für Gastroenterologie und Hepatologie, Medizinische Universität Graz, Graz, Österreich

\title{
Nichtinvasive Diagnostik bei nichtalkoholischer Fettlebererkrankung (NAFLD)
}

\section{Einleitung}

Weltweit spielt die nichtalkoholische Fettlebererkrankung (NAFLD) parallel zur Adipositasepidemie eine zunehmende Rolle. Ihre Prävalenz wird in Europa mit $24 \%$ der Gesamtbevölkerung angegeben [1]. Je nach untersuchter Population haben $7-30 \%$ der NAFLD-Patienten eine nichtalkoholische Steatohepatitis (NASH), die mit einer erhöhten leberbezogenen Mortalität verbunden ist.

Mehrere Studien haben das Vorliegen einer höhergradigen Fibrose/Zirrhose (Fibrosestadium F3 bis F4) als wesentlichen prognostischen Faktor für leberbezogene Mortalität identifiziert [2, 3]. Bei der hohen Prävalenz der NAFLD ist eine generelle bioptische Abklärung nicht machbar; die Entwicklung nichtinvasiver diagnostischer Tests für Steatose bzw. Fibrose ist daher Gegenstand intensiver Forschung $[4,5]$.

\section{Leberbiopsie - Goldstandard der NASH-Diagnostik?}

Trotz großer Fortschritte bei der Entwicklung nichtinvasiver Tests kann die Diagnose NASH aktuell nur histologisch gestellt werden und die Endpunkte klinischer Studien zur NASH-Therapie (Besserung des Fibrosestadiums, Rückbildung von NASH) basieren auf (sequenziellen) Kontrollbiopsien.

Aktualisierung von: Stauber R (2009). Nichtinvasive Diagnose der Leberfibrose bei chronischen Hepatopathien. J Gastroenterol Hepatol Erkr 7(4):12-17.
Leider ist die Leberbiopsie mit einer beträchtlichen Morbidität (postinterventioneller Schmerz in 10-30\%) und einer gewissen Mortalität (ca. 0,1-0,01 \%) verbunden. Darüber hinaus ist die Aussagekraft durch „sampling error“ "bzw. ,interobserver error" eingeschränkt und eine standardisierte Beschreibung der Leberhistologie mit Angabe von validierten Scores [6] in der klinischen Praxis nicht immer gewährleistet.

\section{Einfache Fibrosetests}

Einfache Fibrosetests basieren auf einer Kombination von klinischen Parametern und Routinelaborwerten, die verschiedene Aspekte der Leberfunktion reflektieren. Die Bestimmung ist kostengünstig und die Berechnung wird durch mobile Apps und/oder „website calculators“ im Internet erleichtert.

\section{NAFLD Fibrosis Score}

Angulo et al. untersuchten eine Kohorte von 733 Patienten mit nichtalkoholischer Fettlebererkrankung und entwickelten den NAFLD Fibrosis Score (NFS), der auf Alter, Hyperglykämie, BMI, Thrombozytenzahl, Albumin und AST-ALT-Ratio basiert, als Prädiktor für fortgeschrittene Fibrose (F3 bis F4; [7]).

\section{Fibrosis-4-Index (FIB-4)}

Der FIB-4-Index wurde ursprünglich an einer Kohorte von HIV/HCV-Koinfizierten beschrieben [8], aber mittlerweile so- wohl bei Patienten mit HCV-Monoinfektion als auch NAFLD validiert.

$$
\begin{aligned}
& \text { FIB }-4=(\text { AST } \times \text { Alter }[\text { Jahre }]) / \\
& (\text { Thrombozytenzahl }[\mathrm{G} / 1] \times \sqrt{\text { ALT }})
\end{aligned}
$$

Unter Verwendung von oberen $(>2,67)$ bzw. unteren $(<1,30)$ FIB-4-Grenzwerten für eine hohe bzw. geringe Vortestwahrscheinlichkeit auf eine fortgeschritten Fibrose (F3 bis F4) können ca. 60\% der Patienten mit NAFLD ohne Leberbiopsie korrekt klassifiziert werden [9]. Vergleichende Studien zeigten eine etwas höhere diagnostische Genauigkeit von FIB-4 als NFS [9, 10]. Eine automatische Berechnung und Ausgabe des Werts kann im klinischen Labor relativ einfach implementiert und für die klinische Praxis hilfreich eingesetzt werden.

eLIFT

Französische Autoren entwickelten einen weiteren, auf Routineparametern basierenden Fibrosetest, den „easy liver fibrosis test“ (eLIFT), an einer großen Kohorte von Patienten mit chronischer Lebererkrankung (vorwiegend Hepatitis $\mathrm{C}$ und NAFLD; [11]; • Tab. 1). Für eLIFT (Cutoff-Wert $\geq 8$ Punkte) wurde eine ähnliche Treffsicherheit wie für FIB-4, aber eine höhere Spezifität bei Patienten über 60 Jahre beschrieben. Diese interessanten Daten wurden jedoch noch nicht ausreichend extern validiert.

\section{Hepamet-fibrosis-Score}

Spanische Autoren entwickelten kürzlich den Hepamet-fibrosis-Score (HFS), der 


\begin{tabular}{l|l}
$\begin{array}{l}\text { Tab. } 1 \\
\text { Parameter }\end{array}$ & Pasy liver fibrosis test (eLIFT) \\
\hline $\begin{array}{l}\text { Alter (Jahre) } \\
<40\end{array}$ & 0 \\
$\geq 40$ & 3 \\
\hline Geschlecht & \\
\hline Weiblich & 0 \\
\hline Männlich & 1 \\
\hline AST (U/I) & \\
$<35$ & 0 \\
\hline $35-69$ & 2 \\
\hline$\geq 70$ & 4 \\
\hline$\gamma-G T$ (U/I) & \\
$<35$ & 0 \\
\hline $35-89$ & 1 \\
$\geq 90$ & 2 \\
\hline Thrombozytenzahl(G/l) & \\
\hline$\geq 250$ & 0 \\
\hline $170-249$ & 1 \\
\hline$<170$ & 4 \\
\hline Prothrombinzeit (\%) & \\
\hline$\geq 97$ & 0 \\
$84-96$ & 2 \\
\hline$<84$ & 4 \\
\hline
\end{tabular}

auf Geschlecht, Alter, HOMA-IR, Diabetes, AST, Albumin und Thrombozytenzahl basiert [12]. Für die Berechnung steht ein „website calculator“ zur Verfügung (hepamet-fibrosis-score.eu). Die diagnostische Performance war derjenigen des FIB-4 überlegen (AUROC 0,85 vs. 0,80 für die Diagnose von F3 bis F4). Auch dieser Test bedarf noch weiterer externer Validierung.

\section{Kommerzielle Fibrosetests}

\section{Fibrotest}

Französische Studien identifizierten aus einer großen Zahl von Variablen bei 339 HCV-Patienten ein Panel von 5 Markern ( $\alpha 2-$ Makroglobulin, Haptoglobin, Apolipoprotein A1, GGT und Gesamtbilirubin; [13]), das als Fibrotest ${ }^{\mathrm{Tm}}$ (Biopredictive, Paris, Frankreich) vermarktet wird. Die Berechnung von Fibrotest durch einen patentierten Algorithmus wird vom Hersteller in Rechnung gestellt. Ein Einsatz von Fibrotest bei NAFLD ergab AUROC von 0,74-0,81 für F3 bis $\mathrm{F} 4[14,15]$.

\section{Enhanced Liver Fibrosis (ELF)}

In einer großen europäischen Multizenterstudie an 1021 Patienten mit verschiedenen chronischen Hepatopathien wurde ein Algorithmus basierend auf Hyaluronsäure, PIIINP und TIMP-1 entwickelt [16] und als Enhanced Liver Fibrosis $\left(\mathrm{ELF}^{\mathrm{rx}}\right)$ von Siemens Healthineers (Wien, Österreich) vermarktet. Bei der nichtalkoholischen Fettleber konnte dieser Test eine signifikante bzw. fortgeschrittene Fibrose zuverlässig anzeigen [17]. Mittlerweise wurde der ELF-Test in mehreren NAFLD-Kohorten validiert $[10,18,19]$. Eigene Daten bestätigen die hohe diagnostische Aussagekraft eines Cut-offWerts von 9,8 für F3 bis F4 (PPV 70\%, NPV $90 \%$; [10]).

\section{Fibrometer}

Der Fibrometer-Test basiert auf a2Makroglobulin, Hyaluronsäure, AST, Thrombozytenzahl, Prothrombinzeit, Harnstoff und Alter [20], weiterentwickelt zum FibrometerV2G (Version 2, enthält Geschlecht als zusätzliche Variable; [15, 21]) und FibrometerV3G (Version 3, enthält GGT statt Hyaluronsäure; [22]). Dieser ursprünglich für die Virushepatitis entwickelte Test wurde bei NAFLD mehrfach validiert [10, 15]; seine diagnostische Performance war dem Fibrotest überlegen [15] und dem ELF-Test vergleichbar [10]. Der Fibrometer wird von der Firma Echosens (Paris, Frankreich) - entweder als alleiniger Bluttest oder in Kombination mit Fibroscan (Fibrometer ${ }^{\mathrm{VCTE}}$, siehe unten) - kommerziell angeboten.

\section{Physikalische Methoden (Elastographie)}

Während konventionelle bildgebende Verfahren (Sonographie, CT, MRT) vorwiegend eine fortgeschrittene Zirrhose mit Zeichen der portalen Hypertension erfassen können, ermöglichen neuere Verfahren eine Messung der Lebersteifigkeit (Elastographie) als indirekten Parameter für Leberfibrose.

\section{Transiente Elastographie (Fibroscan)}

Die vibrationskontrollierte transiente Elastographie (VCTE; FibroScan ${ }^{\mathrm{Ts}}$, Echosens, Paris, Frankreich) ist ein Ultraschallverfahren zur Messung der Lebersteifigkeit [23]. Ein perkutan aufgesetzter „transducer“ sendet niederfrequente Vibrationen in das Leberparenchym und aus der Geschwindigkeit der reflektierten Scherwelle wird die Lebersteifigkeit abgeleitet (Werte in $\mathrm{kPa}$ ). Die Messung wird 10-mal wiederholt und ist nur bei geringer Streuung (IQR $<30 \%$ des Medians bei Werten $>7 \mathrm{kPa}$ ) aussagekräftig. Werte von $<7 \mathrm{kPa}$ können generell als verlässlich angesehen werden, da es keine klinische Situation gibt, die die mittels VCTE gemessene Lebersteifigkeit falschniedrig verändert. Jedoch können andererseits falsch-hohe VCTE-Ergebnisse vorliegen (rezente Nahrungsaufnahme, mechanische Cholestase, kardiale Stauung etc.). Im Gegensatz zur Leberbiopsie wird ein relativ großer Leberzylinder von ca. $4 \mathrm{~cm}^{3}$ erfasst. Der Fibroscan ist rasch durchführbar (ca. $5 \mathrm{~min}$ ) und relativ einfach erlernbar, allerdings ist die Messung nicht selten wegen Adipositas oder enger Interkostalräume unmöglich.

Eine französische Studie an 370 gesunden Personen ohne metabolisches Syndrom ergab einen Normbereich von $5,3 \pm 1,5 \mathrm{kPa}$ [24]. $\mathrm{Zu}$ beachten ist, dass die Cut-off-Werte je nach Ätiologie der Lebererkrankung variieren (• Abb. 1). Wichtig ist auch die Wahl der richtigen Sonde: $\mathrm{m}$-Sonde für Patienten mit BMI $<30$ und XL-Sonde für Patienten mit $\mathrm{BMI} \geq 30$. Bei höhergradiger Adipositas ist die Messung trotzdem nicht selten unmöglich.

Nach Wong et al. besitzt der Fibroscan auch bei NAFLD hohe Treffsicherheit, als Cut-off-Wert für eine höhergradige Fibrose (F3 bis F4) wurde $8,7 \mathrm{kPa}$ angegeben [25]. Eine rezente Studie an einer großen britischen Kohorte kam zu ähnlichen Daten bei etwas höheren Cut-off-Werten $(\mathrm{F} \geq 2: 8,2 \mathrm{kPa} ; \mathrm{F} \geq 3: 9,7 \mathrm{kPa} ; \mathrm{F} 4: 13,6 \mathrm{kPa}$; $[26,27])$. Anhand eigener Daten konnten wir hingegen $11,0 \mathrm{kPa}$ als optimalen Cut-off-Wert für F3 bis F4 identifizieren [10]. Diese Unterschiede sind vorwiegend durch unterschiedliche Verteilung 
Hier steht eine Anzeige.

\section{曾 Springer}


der Fibrosestadien in den verschiedenen Kohorten zu erklären („selection bias“).

\section{MR-Elastographie}

Die MR-Elastographie (MRE) bietet den Vorteil, größere Leberabschnitte zu erfassen, und liefert die höchste Genauigkeit unter allen nichtinvasiven Fibrosediagnostika [28]. Nachteil ist das dafür benötigte spezielle Equipment, die relativ aufwändige Durchführung und - damit verbunden - die limitierte Verfügbarkeit. Eine prospektive Studie an 117 Patienten mit bioptisch verifizierter NAFLD zeigte eine hohe diagnostische Genauigkeit für F3 bis F4 bei einem Cut-off-Wert von $>3,63 \mathrm{kPa}$ [29]. Im direkten Vergleich zum Fibroscan war die MRE überlegen (AUROC 0,87 vs. 0,80 für F3 bis F4; [30]).

\section{Kombinierte Fibrosetests}

Eine Kombination von blutbasierten Fibrosetests mit Fibroscan kann die diagnostische Genauigkeit erwartungsgemäß verbessern. Ein vielversprechender Test, vermarktet vom Fibroscan-Hersteller Echosens, ist der Fibrometer ${ }^{\mathrm{VCTE}}$, der ein genaueres Staging der Fibrose sowohl vs. Fibroscan alleine als auch vs. Fibrometer alleine zeigte [31].

\section{Vergleichende Bewertung verschiedener nichtinvasiver Fibrosetests}

In -Tab. 2 findet sich ein Überblick der diagnostischen Genauigkeit verschiedener nichtinvasiver Fibrosetests anhand von ROC-curve-Analysen einer direkten Vergleichsstudie in einer österreichischen Kohorte von 186 NAFLDPatienten [10]. Dabei zeigte sich eine relativ gute Treffsicherheit des einfachen, auf Routineparametern basierenden FIB-4. Unter den kommerziellen Fibrosepanels zeigten der ELF-Test und der Fibrometer ${ }^{\mathrm{V} G / \mathrm{V} 3 \mathrm{G}}$ die beste Performance. Der FibroScan war in der „Perprotocol“-Analyse (wenn nur zuverlässige Werte herangezogen werden) den anderen Methoden überlegen, nicht jedoch in einer „Intent-to-diagnose“-Analyse (wenn auch unzuverlässige Werte herangezogen und misslungene Unter-

J. Gastroenterol. Hepatol. Erkr. 2019 · 17:75-81 https://doi.org/10.1007/s41971-019-00058-1 (c) Der/die Autor(en) 2019

\section{R. Stauber \\ Nichtinvasive Diagnostik bei nichtalkoholischer Fettlebererkrankung (NAFLD)}

\section{Zusammenfassung}

Die nichtalkoholische Fettlebererkrankung (NAFLD) ist weltweit sehr häufig, ihre Prävalenz in Europa wurde mit $24 \%$ der Bevölkerung angegeben. Dabei zeigen 10-30\% der Patienten einen progredienten Verlauf, der auch als nichtalkoholische Steatohepatitis (NASH) bezeichnet wird. Das Vorliegen einer höhergradigen Lebervernarbung (Fibrose, F3/Zirrhose, F4) ist ein wichtiger Prognosefaktor mit erhöhter leberbezogener Mortalität und Therapiebedürftigkeit. Die histologische Befundung von Leberbiopsien gilt derzeit als Referenzmethode für die Diagnose einer NASH und die Bestimmung des Fibrosestadiums. Aufgrund der hohen Prävalenz von NAFLD ist eine möglichst genaue nichtinvasive Risikostratifizierung notwendig, um betroffene Patienten für die Leberbiopsie zu identifizieren. Unter den blutbasierten nichtinvasiven Fibrosetests („blood fibrosis tests") gibt es a) einfache Tests (auf Routineparametern basierend) wie den NAFLD-fibrosis-Score (NFS) und den Fibrosis-4-Index (FIB-4) sowie b) kom- merzielle Fibrosetests wie Enhanced-liverfibrosis(ELF $\left.{ }^{\mathrm{TM}}\right)$-Test (Siemens Healthineers, Wien, Österreich), FibroTest ${ }^{\mathrm{TM}}$ (Biopredictive, Paris, Frankreich) und FibroMeter (Echosens, Paris, Frankreich). Bei den ultraschallbasierten Verfahren ist die transiente Elastographie ("vibration-controlled transient elastography", VCTE; FibroScan [VCTE; FibroScan ${ }^{\mathrm{TM}}$, Echosens, Paris, Frankreich]) breit verfügbar, während die MR-Elastographie (MRE) selten verwendet wird, aber wahrscheinlich genauere Resultate liefert. In direkten Vergleichsstudien zeigten kommerzielle Blutfibrosetests, VCTE und MRE die höchste diagnostische Genauigkeit bei der nichtinvasiven Einschätzung des Fibrosestadiums bei NAFLD. Unter den einfachen Tests eignet sich FIB-4 sehr gut zum Ausschluss einer höhergradigen Fibrose und bietet sich damit für ein breites Screening (z. B. bei Patienten mit Diabetes) an.

\section{Schlüsselwörter}

Signifikante Fibrose · Fortgeschrittene Fibrose - Zirrhose - Biomarker - Elastographie

\section{Noninvasive Diagnostics in Nonalcoholic Fatty Liver Disease (NAFLD)}

\section{Abstract}

Nonalcoholic fatty liver disease (NAFLD) is highly prevalent worldwide, its prevalence in Europe has been estimated at $24 \%$ of the population. Among patients with NAFLD, $10-30 \%$ show the progressive form, nonalcoholic steatohepatitis (NASH), and presence of advanced fibrosis (F3)/cirrhosis (F4) has been identified as an important prognostic factor indicating an increased risk for liver-related mortality. Histological evaluation of liver biopsies is currently considered the gold standard for diagnosis of NASH and for staging hepatic fibrosis but, due to the high prevalence of NAFLD, noninvasive risk stratification is needed to select patients for liver biopsy. Bloodbased noninvasive fibrosis tests comprise (a) simple tests (based on routine clinical and laboratory parameters) such as the NAFLD fibrosis score (NFS) and the fibrosis- 4 index (FIB-4) and (b) commercial fibrosis panels such as the enhanced liver fibrosis (ELF) test (Siemens Healthineers, Wien, Österreich), FibroTest $^{\mathrm{TM}}$ (Biopredictive, Paris, Frankreich), and FibroMeter (Echosens, Paris, Frankreich). Among physical methods, vibrationcontrolled transient elastography (VCTE; FibroScan $^{\mathrm{T}}{ }^{\mathrm{M}}$, Echosens, Paris, Frankreich) is widely available, while MR elastography (MRE) is rarely used but seems to provide very accurate results. In comparative studies, commercial fibrosis panels, VCTE and MRE showed superior diagnostic accuracy for the noninvasive diagnosis of fibrosis stage in patients with NAFLD. Among the simple tests, FIB-4 can sufficiently exclude advanced fibrosis and seems thus well suited for broad testing, e.g., in patients with diabetes.

\section{Keywords}

Significant fibrosis - Advanced fibrosis . Cirrhosis - Biomarkers · Elastography 


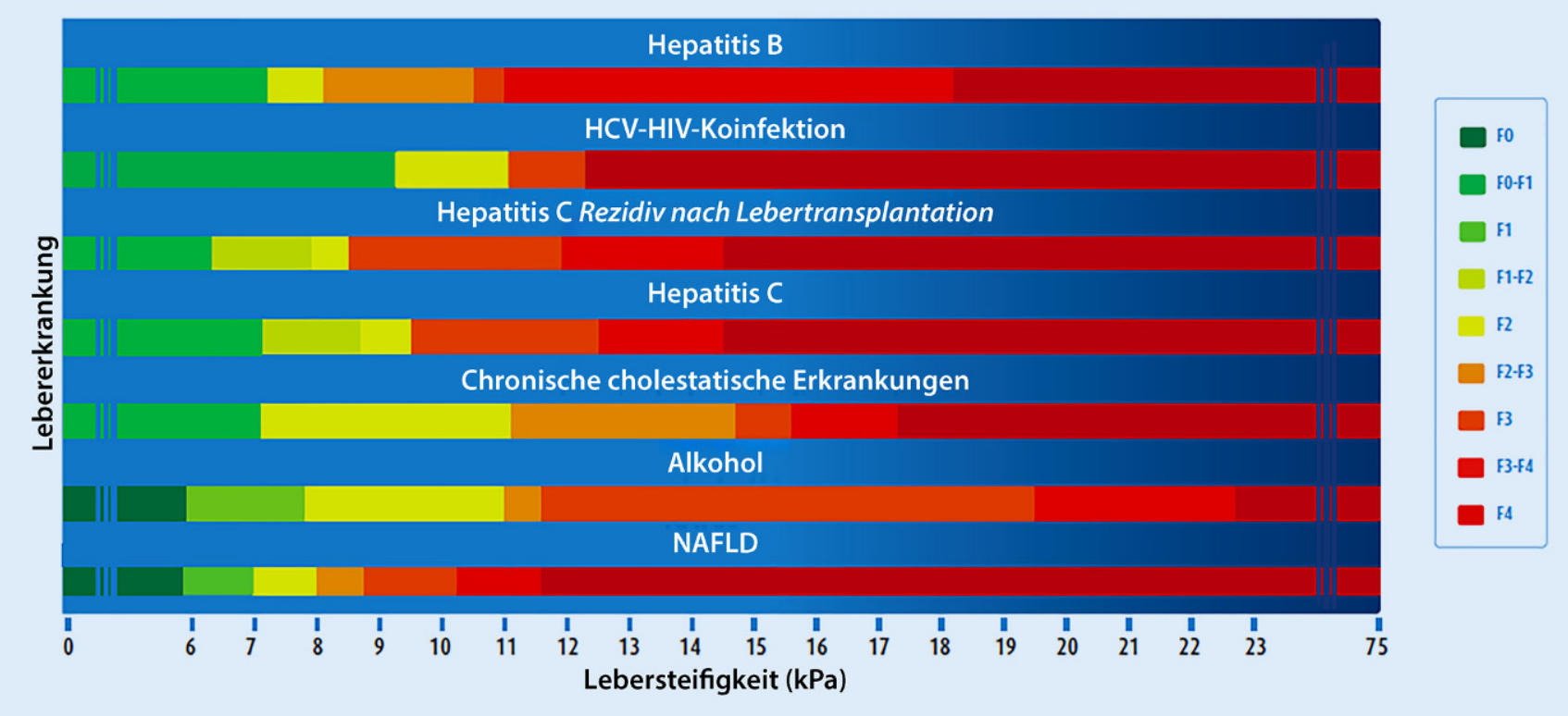

Abb. 1 A Grenzwerte zur nichtinvasiven Einschätzung von Fibrosestadien anhand der mit VCTE gemessenen Leberelastizität je nach Ätiologie der Lebergrunderkrankung. (@ Echosens ${ }^{\mathrm{T}}$, Paris, Frankreich)

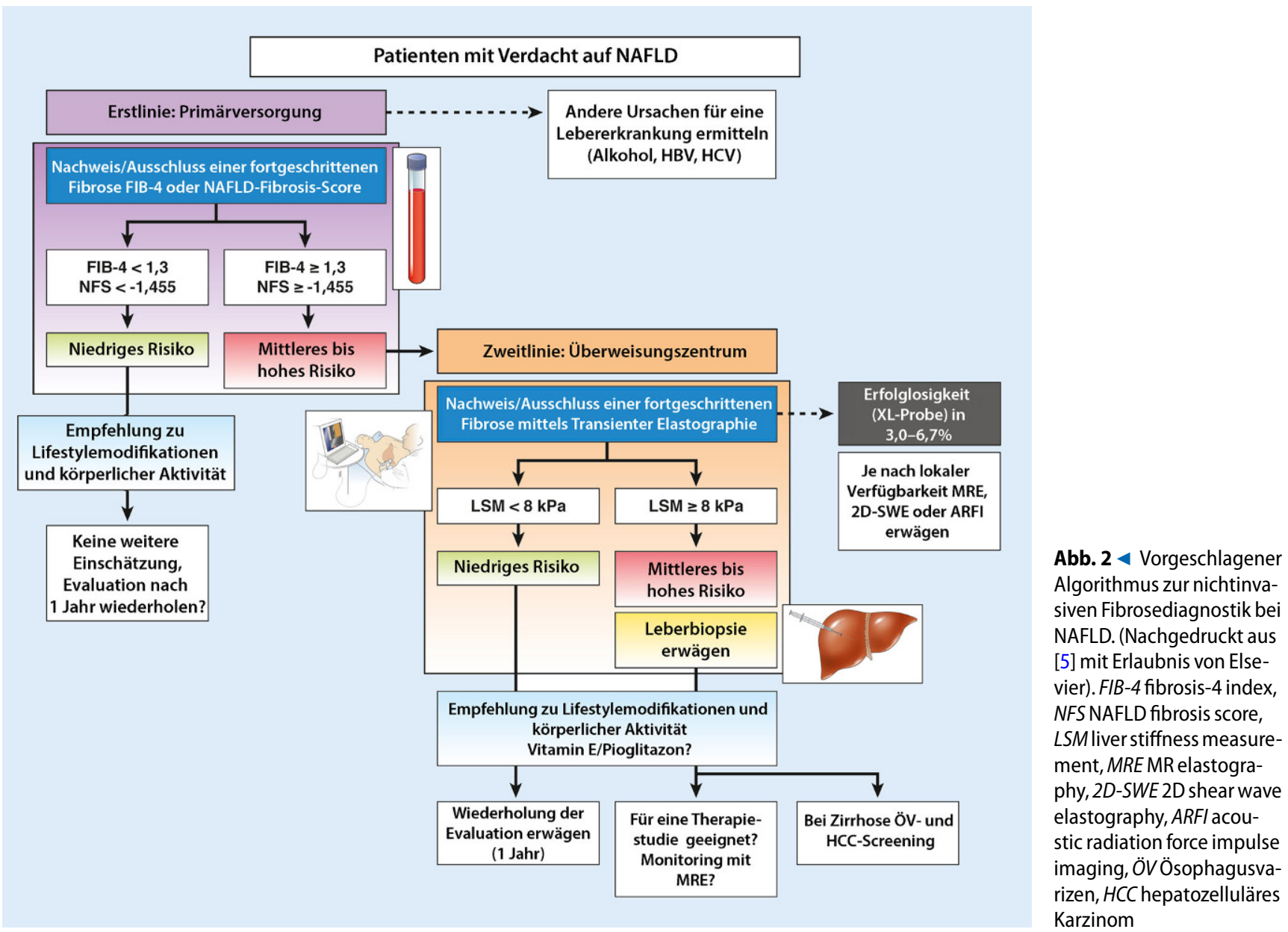


Tab. 2 Diagnostische Wertigkeit von nichtinvasiven Fibrosetests bei NAFLD. Vergleichsstudie von 6 verschiedenen Fibrosetests an einer österreichischen NAFLD-Kohorte $(n=186)$. (Modifiziert nach Staufer et al. [10])

\begin{tabular}{|c|c|c|c|c|}
\hline Test & AUROC F $\geq 2$ & AUROC F $\geq 3$ & AUROC F $\geq 4$ & AUROC F $\geq 3 \&$ NASH \\
\hline FIB-4 & 0,80 & 0,82 & 0,86 & 0,81 \\
\hline NFS & 0,78 & 0,80 & 0,79 & 0,79 \\
\hline ELF & 0,85 & 0,90 & 0,92 & 0,90 \\
\hline Fibrometer V2G & 0,86 & 0,88 & 0,95 & 0,89 \\
\hline Fibrometer V3G & 0,84 & 0,88 & 0,94 & 0,88 \\
\hline LSM & 0,85 & 0,91 & 0,95 & 0,87 \\
\hline LSM „per protocol“ & 0,87 & 0,95 & 0,98 & 0,91 \\
\hline
\end{tabular}

suchungen als falsch-positiv bzw. falschnegativ gewertet werden).

\section{Quantifizierung des Leberfettgehalts}

Histologisch wird der Leberfettgehalt routinemäßig semiquantitativ angegeben (Prozentsatz der verfetteten Leberzellen) und üblicherweise in 4 Klassen eingeteilt (S0: 0-5\%, S1: 5-33\%, S2: 33-66\%, S3: $>66 \%)$. Eine morphometrische Quantifizierung erlaubt eine genauere Bestimmung des Leberfettgehalts, wird aber aufgrund der aufwändigen Messung nur selten durchgeführt.

Der „controlled attenuation parameter" (CAP) des FibroScan beruht auf Abschwächung des Ultraschallsignals durch Fett und erlaubt eine nichtinvasive Quantifizierung des Leberfettgehalts mit Cut-off-Werten von $302 \mathrm{~dB} / \mathrm{m}$ für $\mathrm{S} \geq 1,331 \mathrm{~dB} / \mathrm{m}$ für $\mathrm{S} \geq 2$ und $337 \mathrm{~dB} / \mathrm{m}$ für S3 [26].

Die MR-basierte Messung des Leberfettgehalts mittels "proton density fat fraction" (PDFF) ist im Vergleich zur MRE relativ einfach durchzuführen und ermöglicht eine sehr genaue Abschätzung des Leberfettgehalts [28]. Die MR-PDFF war in einer prospektiven Vergleichsstudie dem CAP deutlich überlegen [30].

\section{Fazit für die Praxis}

Die Hauptvorteile nichtinvasiver Fibrosetests liegen im fehlenden Risiko für den Patienten, in der Minimierung des "sampling errors" und in der leichten Wiederholbarkeit bei Verlaufskontrol- len. Einfache Tests wie der Fibrosis-4 Index (FIB-4) eignen sich gut für das Screening von Risikopopulationen, z. B. bei Patienten mit Diabetes, wobei ein FIB-4 $<1,30$ eine höhergradige Leberfibrose recht zuverlässig ausschließen kann (NPV $90 \%$ ). Patienten mit FIB-4 über diesem Grenzwert eignen sich für eine weiterführende Diagnostik im Referenzzentrum mittels Fibroscan und/ oder kommerziellen blutbasierten Fibrosetests wie dem Enhanced Liver Fibrosis (ELF)-Test. Werden dabei bestimmte Cut-off-Werte überschritten (z. B. 9,7 kPa im Fibroscan oder ein ELFScore von 9,8), besteht eine hohe Wahrscheinlichkeit für NASH mit höhergradiger Fibrose und eine weiterführende Leberbiopsie ist empfehlenswert. Ein ähnlicher Algorithmus wurde kürzlich von Castera et al. [5] vorgeschlagen (०Abb. 2).

Solche nichtinvasiven Algorithmen bewähren sich u.a. beim „case finding“ für klinische Studien zur NASH-Therapie. Als therapeutische Konsequenz steht derzeit die Lifestylemodifikation mit Zunahme der körperlichen Aktivität und Gewichtsreduktion im Vordergrund; künftig ist mit der Zulassung neuer medikamentöser Therapieoptionen zu rechnen. Weitere Studien sind notwendig, um den Stellenwert von nichtinvasiven Fibrosetests zur Verlaufskontrolle bei NASH bzw. von therapeutischen Interventionen zu beurteilen.

\section{Korrespondenzadresse}

Rudolf Stauber

Univ.-Klinische Abteilung für Gastroenterologie und Hepatologie, Medizinische Universität Graz Auenbruggerplatz 15, $8036 \mathrm{Graz}$, Österreich rudolf.stauber@medunigraz.at

Funding. Open access funding provided by Medical University of Graz.

\section{Einhaltung ethischer Richtlinien}

Interessenkonflikt. R. Stauber gibt an, dass kein Interessenkonflikt besteht.

Für diesen Beitrag wurden von den Autoren keine Studien an Menschen oder Tieren durchgeführt. Für die aufgeführten Studien gelten die jeweils dort angegebenen ethischen Richtlinien.

Open Access. Dieser Artikel wird unter der Creative Commons Namensnennung 4.0 International Lizenz (http://creativecommons.org/licenses/by/4.0/deed. de) veröffentlicht, welche die Nutzung, Vervielfältigung, Bearbeitung, Verbreitung und Wiedergabe in jeglichem Medium und Format erlaubt, sofern Sie den/die ursprünglichen Autor(en) und die Quelle ordnungsgemäßnennen, einen Link zur Creative Commons Lizenz beifügen und angeben, ob Änderungen vorgenommen wurden.

\section{Literatur}

1. Younossi ZM, Koenig $A B$, Abdelatif $D$, Fazel $Y$, Henry L, Wymer M (2016) Global epidemiology of nonalcoholic fatty liver disease-Meta-analytic assessment of prevalence, incidence, and outcomes. Hepatology 64:73-84

2. Angulo $P$, Kleiner DE, Dam-Larsen $S$, Adams LA, Bjornsson ES, Charatcharoenwitthaya $P$, Mills $P R$ et al (2015) Liver fibrosis, but no other Histologic features, is associated with long-term outcomes of patients with nonalcoholic fatty liver disease. Gastroenterology 149:389-397e10

3. Ekstedt $M$, Hagstrom $H$, Nasr P, Fredrikson $M$, Stal P, Kechagias S, Hultcrantz R (2015) Fibrosis stage is the strongest predictor for disease-specific mortality in NAFLD after up to 33 years of followup. Hepatology 61:1547-1554

4. Younossi ZM, Loomba R, Anstee QM, Rinella ME, Bugianesi E, Marchesini G, Neuschwander-Tetri BA et al (2018) Diagnostic modalities for nonalcoholic fatty liver disease, nonalcoholic steatohepatitis, and associated fibrosis. Hepatology 68:349-360

5. Castera L, Friedrich-Rust M, Loomba R (2019) Noninvasive assessment of liver disease in patients with nonalcoholic fatty liver disease. Gastroenterology 156:1264-1281e4

6. Kleiner DE, Brunt EM, Van Natta M, Behling C, Contos MJ, Cummings OW, Ferrell LD et al (2005) Design and validation of a histological scoring system for nonalcoholic fatty liver disease. Hepatology 41:1313-1321

7. Angulo P, Hui JM, Marchesini G, Bugianesi E, George J, Farrell GC, Enders F et al (2007) The NAFLD fibrosis score: a noninvasive system that 
identifies liver fibrosis in patients with NAFLD. Hepatology 45:846-854

8. Sterling RK, Lissen E, Clumeck N, Sola R, Correa MC, Montaner J et al (2006) Development of a simple noninvasive index to predict significant fibrosis in patients with HIV/HCV coinfection. Hepatology 43:1317-1325

9. McPherson S, Stewart SF, Henderson E, Burt AD, Day CP (2010) Simple non-invasive fibrosis scoring systems can reliably exclude advanced fibrosis in patients with non-alcoholic fatty liver disease. Gut 59:1265-1269

10. Staufer K, Halilbasic E, Spindelboeck W, Eilenberg M, Prager G, Stadlbauer V, Posch A, Munda P Marculescu R, Obermayer-Pietsch B, Stift J, Lackner C, Trauner M, Stauber RE (2019) Evaluation and comparison of six noninvasive tests for prediction of significant or advanced fibrosis in nonalcoholic fatty liver disease. UEG J. https://doi.org/10.1177/ 2050640619865133

11. Boursier J, de Ledinghen V, Leroy V, Anty R, Francque S, Salmon D, Lannes A et al (2017) A stepwise algorithm using an at-a-glance first-line test for the non-invasive diagnosis of advanced liver fibrosis and cirrhosis. J Hepatol 66:1158-1165

12. Ampuero J, Pais $R$, Aller $R$, Gallego-Duran $R$ Crespo J, Garcia-Monzon C, Boursier J et al (2019) Development and validation of hepamet fibrosis scoring system a simple, noninvasive test to identify patients with nonalcoholic fatty liver disease with advanced fibrosis. Clin Gastroentero Hepatol. https://doi.org/10.1016/j.cgh.2019.05. 051

13. Imbert-Bismut F, Ratziu V, Pieroni L, Charlotte F, Benhamou Y, Poynard T (2001) Biochemical markers of liver fibrosis in patients with hepatitis $C$ virus infection: a prospective study. Lancet 357:1069-1075

14. Ratziu V, Massard J, Charlotte F, Messous D, Imbert-Bismut $\mathrm{F}$, Bonyhay $\mathrm{L}$, Tahiri $\mathrm{M}$ et al (2006) Diagnostic value of biochemical markers (FibroTest-FibroSURE) for the prediction of liver fibrosis in patients with non-alcoholic fatty liver disease. BMC Gastroenterol 6:6

15. Boursier J, Vergniol J, Guillet A, Hiriart JB, Lannes A, Le Bail B, Michalak S (2016) et al Diagnostic accuracy and prognostic significance of blood fibrosis tests and liver stiffness measurement by FibroScan in non-alcoholic fatty liver disease. JHepatol 65:570-578

16. Rosenberg WM, Voelker M, Thiel R, Becka M, Burt A, Schuppan D, Hubscher Setal (2004) Serum markers detect the presence of liver fibrosis: a cohort study. Gastroenterology 127:1704-1713

17. Guha IN, Parkes J, Roderick P, Chattopadhyay D, Cross R, Harris S, Kaye P et al (2008) Noninvasive markers of fibrosis in nonalcoholic fatty liver disease: Validating the European Liver Fibrosis Panel and exploring simple markers. Hepatology 47:455-460

18. Lichtinghagen $R$, Pietsch $D$, Bantel $H$, Manns MP, Brand K, Bahr MJ (2013) The Enhanced Liver Fibrosis (ELF) score: normal values, influence factors and proposed cut-off values. J Hepatol 59:236-242

19. Fagan KJ, Pretorius $\mathrm{CJ}$, Horsfall LU, Irvine KM, Wilgen U, Choi K, Fletcher LM et al (2015) ELF score $\rangle /=9.8$ indicates advanced hepatic fibrosis and is influenced by age, steatosis and histological activity. Liver Int 35:1673-1681

20. Cales P, Oberti F, Michalak S, Hubert-Fouchard I, Rousselet MC, Konate A, Gallois Y et al (2005) A novel panel of blood markers to assess the degree of liver fibrosis. Hepatology 42:1373-1381
21. Leroy $V$, Halfon $P$, Bacq Y, Boursier J, Rousselet MC, Bourliere M, de Muret A et al (2008) Diagnostic accuracy, reproducibility and robustness of fibrosis blood tests in chronic hepatitis C: a meta-analysis with individual data. Clin Biochem 41:1368-1376

22. Cales $P$, Boursier J, Bertrais $S$, Oberti F, Gallois $Y$ Fouchard-Hubert I, Dib N et al (2010) Optimization and robustness of blood tests for liver fibrosis and cirrhosis. Clin Biochem 43:1315-1322

23. Sandrin L, Fourquet B, Hasquenoph JM, Yon $S$, Fournier C, Mal F, Christidis Cet al (2003) Transient elastography: a new noninvasive method for assessment of hepatic fibrosis. Ultrasound Med Biol 29:1705-1713

24. Roulot D, Czernichow S, Le Clesiau H, Costes JL, Vergnaud AC, Beaugrand M (2008) Liver stiffness values in apparently healthy subjects: influence of gender and metabolic syndrome. J Hepatol 48:606-613

25. Wong VW, Vergniol J, Wong GL, Foucher J, Chan HL, Le Bail B, Choi PC et al (2010) Diagnosis of fibrosis and cirrhosis using liver stiffness measurement in nonalcoholic fatty liver disease. Hepatology 51:454-462

26. Eddowes PJ, Sasso $M$, Allison $M$, Tsochatzis $E$ Anstee QM, Sheridan D, Guha IN et al (2019) Accuracy of fibroscan controlled attenuation parameter and liver stiffness measurement in assessing steatosis and fibrosis in patients with nonalcoholic fatty liver disease. Gastroenterology 156:1717-1730

27. Siddiqui MS, Vuppalanchi R, Van Natta ML, Hallinan E, Kowdley KV, Abdelmalek M, NeuschwanderTetri BA et al (2019) Vibration-controlled transient elastography to assess fibrosis and steatosis in patients with nonalcoholic fatty liver disease. Clin Gastroenterol Hepatol 17:156-163e2

28. Dulai PS, Sirlin CB, Loomba R (2016) MRI and MRE for non-invasive quantitative assessment of hepatic steatosis and fibrosis in NAFLD and NASH: clinical trials to clinical practice. J Hepatol 65:1006-1016

29. Loomba R, Wolfson T, Ang B, Hooker J, Behling C, Peterson M, Valasek M et al (2014) Magnetic resonance elastography predicts advanced fibrosis in patients with nonalcoholic fatty liver disease: a prospective study. Hepatology 60:1920-1928

30. Park CC, Nguyen P, Hernandez C, Bettencourt $R$ RamirezK, Fortney L, Hooker Jetal (2017) Magnetic resonance elastography vs transient elastography in detection of fibrosis and noninvasive measurement of steatosis in patients with biopsy-proven nonalcoholic fatty liver disease. Gastroenterology 152:598-607.e2

31. Ducancelle A, Leroy V, Vergniol J, Sturm N, Le Bail B, Zarski JP, Nguyen Khac E et al (2017) A single test combining blood markers and elastography is more accurate than other fibrosis tests in the main causes of chronic liver diseases. JClin Gastroenterol 51:639-649

Hinweis des Verlags. Der Verlag bleibt in Hinblick auf geografische Zuordnungen und Gebietsbezeichnungen in veröffentlichten Karten und Institutsadressen neutral.

\section{A. U. Dignass, P. Esters, H. Schulze (Hrsg.) Biologische Therapie der chronisch entzündlichen Darmerkrankungen}

Bremen: UNI-MED Science 2019, 3. Auflage, 79 S., 12 Abb., (ISBN: 978-3 8374-1571-1), Hardcover 29,80 EUR

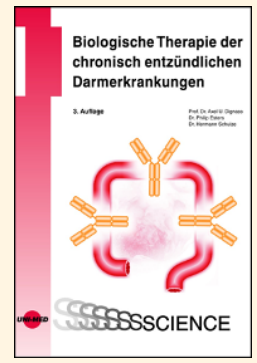

Soeben erschienen ist die dritte Auflage des Kurzbuches „Biologische Therapien der chronisch entzündlichen Darmerkrankungen", verfasst von einem der führenden deutschen und internationalen Experten auf dem Gebiet, Prof. Dr. med. Axel Dignass.

Dieses Buch stellt in aller Kürze, aber dennoch mit den notwendigen Details sowohl die epidemiologischen, ätiopathogenetischen und klassischen Therapieprinzipien der chronisch entzündlichen Darmerkran kungen zusammen. Zugleich bietet es eine sich auf der Höhe der Zeit befindliche Übersicht über die Möglichkeiten der biologischen Therapie bei chronisch entzündlichen Darmerkrankungen, inklusive der aktuellen Studienlage, aber auch der klinisch praktischen Anwendung der uns heute zur Verfügung stehenden biologischen Substanzen.

Dabei ergehen sich die Autoren nicht in unnötigen Details, sondern präsentieren die für die klinische Entscheidung relevanten Daten in übersichtlicher Form. Sie bieten aber auch einen praktischen Leitfaden, welcher für die initiale oder auch sequentielle Anwendung der inzwischen vielen Substanzen in unterschiedlichen $\mathrm{kli}$ nischen Situationen gut verwendet werden kann. Darüber hinaus bietet das Buch praktische Hilfe im begleitenden Management der Patienten sowie im Nebenwirkungsmanagement der Biologika.

Das Buch ist möglicherweise für absolute Spezialfragen für den CED-Spezialisten zu wenig detailliert ausgeführt, für den klinisch breit tätigen Gastroenterologen stellt es jedoch ein wertvolles und gut referenziertes Nachschlagwerk dar.

Markus Peck-Radosavljevic, Klagenfurt 